\title{
DECOMPOSITIONS OF COMPLETE BIPARTITE GRAPHS AND COMPLETE GRAPHS INTO PATHS, STARS, AND CYCLES WITH FOUR EDGES EACH
}

\author{
TAY-WoeI ShyU \\ Division of Preparatory Programs for Overseas Chinese Students \\ National Taiwan Normal University \\ New Taipei City 24449, Taiwan, R.O.C. \\ e-mail: twhsu@ntnu.edu.tw
}

\begin{abstract}
Let $G$ be either a complete graph of odd order or a complete bipartite graph in which each vertex partition has an even number of vertices. In this paper, we determine the set of triples $(p, q, r)$, with $p, q, r>0$, for which there exists a decomposition of $G$ into $p$ paths, $q$ stars, and $r$ cycles, each of which has 4 edges.
\end{abstract}

Keywords: complete graph, complete bipartite graph, path, star, cycle, decomposition.

2010 Mathematics Subject Classification: 05C38, 05C51.

\section{REFERENCES}

[1] A. Abueida and M. Daven, Multidesigns for graph-pairs of order 4 and 5, Graphs Combin. 19 (2003) 433-447.

doi:10.1007/s00373-003-0530-3

[2] A. Abueida and M. Daven, Multidecompositons of the complete graph, Ars Combin. 72 (2004) 17-22.

[3] A. Abueida and T. O'Neil, Multidecomposition of $\lambda K_{m}$ into small cycles and claws, Bull. Inst. Combin. Appl. 49 (2007) 32-40.

[4] A. Abueida and M. Daven, Multidecompositions of several graph products, Graphs Combin. 29 (2013) 315-326.

doi:10.1007/s00373-011-1127-x

[5] A. Abueida and C. Lian, On the decompositions of complete graphs into cycles and stars on the same number of edges, Discuss. Math. Graph Theory 34 (2014) 113125 .

doi:10.7151/dmgt.1719 
[6] B. Alspach and H. Gavlas, Cycle decompositions of $K_{n}$ and $K_{n}-I$, J. Combin. Theory Ser. B 81 (2001) 77-99.

doi:10.1006/jctb.2000.1996

[7] F. Beggas, M. Haddad and H. Kheddouci, Decomposition of complete multigraphs into stars and cycles, Discuss. Math. Graph Theory 35 (2015) 629-639. doi:10.7151/dmgt.1820

[8] A. Bouchet and J. L. Fouquet, Trois types de décomposition d'un graphe en chaînes, Ann. Discrete Math. 17 (1983) 131-141.

[9] D.A. Bryant, S. El-Zanati, C.V. Eyden and D.G. Hoffman, Star decompositions of cubes, Graphs Combin. 17 (2001) 55-59. doi:10.1007/s003730170054

[10] D. Bryant and C.A. Rodger, Cycle Decompositions, in: The CRC Handbook of Combinatorial Designs, 2nd Edition, C.J. Colbourn and J.H. Dinitz (Ed(s)), (CRC Press, Boca Raton, 2007) 373-382.

[11] C.-M. Fu, Y.-L. Lin, S.-W. Lo and Y.-F. Hsu, Decomposition of complete graphs into triangles and claws, Taiwanese J. Math. 18 (2014) 1563-1581. doi:10.11650/tjm.18.2014.3169

[12] K. Heinrich, Path-decompositions, Le Matematiche 47 (1992) 241-258.

[13] K. Heinrich, J. Liu and M. Yu, $P_{4}$-decomposition of regular graphs, J. Graph Theory 31 (1999) 135-143. doi:10.1002/(SICI)1097-0118(199906)31:2〈135::AID-JGT6〉3.0.CO;2-I

[14] M.S. Jacobson, M. Truszczyński and Zs. Tuza, Decompositions of regular bipartite graphs, Discrete Math. 89 (1991) 17-27. doi:10.1016/0012-365X(91)90396-J

[15] S. Jeevadoss and A. Muthusamy, Decomposition of complete bipartite graphs into paths and cycles, Discrete Math. 331 (2014) 98-108. doi:10.1016/j.disc.2014.05.009

[16] S. Jeevadoss and A. Muthusamy, Decomposition of complete bipartite multigraphs into paths and cycles having $k$ edges, Discuss. Math. Graph Theory 35 (2015) $715-731$. doi:10.7151/dmgt.1830

[17] A. Kotzig, From the theory of finite regular graphs of degree three and four, Casopis Pĕst. Mat. 82 (1957) 76-92.

[18] C.S. Kumar, On P4-decomposition of graphs, Taiwanese J. Math. 7 (2003) 657-664. doi:10.11650/twjm/1500407584

[19] H.-C. Lee, M.-J. Lee and C. Lin, Isomorphic path decompositions of $\lambda K_{n, n, n}$ $\left(\lambda K_{n, n, n}^{*}\right)$ for odd $n$, Taiwanese J. Math. 13 (2009) 393-402. doi:10.11650/twjm/1500405344

[20] H.-C. Lee, Multidecompositions of complete bipartite graphs into cycles and stars, Ars Combin. 108 (2013) 355-364. 
[21] H.-C. Lee and J.-J. Lin, Decomposition of the complete bipartite graph with a 1factor removed into cycles and stars, Discrete Math. 313 (2013) 2354-2358. doi:10.1016/j.disc.2013.06.014

[22] H.-C. Lee and Y.-P. Chu, Multidecompositions of the balanced complete bipartite graph into paths and stars, ISRN Combinatorics (2013), Article ID: 398473.

[23] H.-C. Lee, Decomposition of the complete bipartite multigraph into cycles and stars, Discrete Math. 338 (2015) 1362-1369.

doi:10.1016/j.disc.2015.02.019

[24] J.-J. Lin, Decompositions of multicrowns into cycles and stars, Taiwanese J. Math. 19 (2015) 1261-1270. doi:10.11650/tjm.19.2015.3460

[25] C. Lin, J.-J. Lin and T.-W. Shyu, Isomorphic star decomposition of multicrowns and the power of cycles, Ars Combin. 53 (1999) 249-256.

[26] J.-J. Lin and M.-J. Jou, $\left\{C_{k}, P_{k}, S_{k}\right\}$-decompositions of balanced complete bipartite Graphs, Open J. Discrete Math. 6 (2016) 174-179. doi:10.4236/ojdm.2016.63015

[27] C.C. Lindner and C.A. Rodger, Decomposition in Cycles II: Cycle Systems, in: Contemporary Design Theory: A Collection of Surveys, J.H. Dinitz and D.R. Stinson $($ Ed(s)), (New York, Wiley \& Sons, Inc., 1992) 325-369.

[28] C.A. Parker, Complete Bipartite Graph Path Decompositions, Ph.D. Dissertation (Auburn University, Auburn, Alabama, 1998).

[29] H.M. Priyadharsini and A. Muthusamy, $\left(G_{m}, H_{m}\right)$-multifactorization of $\lambda K_{m}$, J. Combin. Math. Combin. Comput. 69 (2009) 145-150.

[30] H.M. Priyadharsini and A. Muthusamy, $\left(G_{m}, H_{m}\right)$-multifactorization of $K_{m, m}(\lambda)$, Bull. Inst. Combin. Appl. 66 (2012) 42-48.

[31] M. Šajna, Cycle decompositions III: complete graphs and fixed length cycles, J. Combin. Des. 10 (2002) 27-78. doi: $10.1002 /$ jcd. 1027

[32] T.-W. Shyu, Decomposition of complete graphs into paths and stars, Discrete Math. 310 (2010) 2164-2169. doi:10.1016/j.disc.2010.04.009

[33] T.-W. Shyu, Decompositions of complete graphs into paths and cycles, Ars Combin. 97 (2010) 257-270.

[34] T.-W. Shyu, Decomposition of complete graphs into paths of length three and triangles, Ars Combin. 107 (2012) 209-224.

[35] T.-W. Shyu, Decomposition of complete graphs into cycles and stars, Graphs Combin. 29 (2013) 301-313. doi:10.1007/s00373-011-1105-3 
[36] T.-W. Shyu, Decomposition of complete bipartite graphs into paths and stars with same number of edges, Discrete Math. 313 (2013) 865-871.

doi:10.1016/j.disc.2012.12.020

[37] T.-W. Shyu, Decomposition of complete bipartite digraphs and complete digraphs into directed paths and directed cycles of fixed even length, Graphs Combin. 31 (2015) $1715-1725$.

doi:10.1007/s00373-014-1442-0

[38] D. Sotteau, Decomposition of $K_{m, n}\left(K_{m, n}^{*}\right)$ into cycles (circuits) of length $2 k$, J. Combin. Theory Ser. B 30 (1981) 75-81.

doi:10.1016/0095-8956(81)90093-9

[39] M. Tarsi, Decomposition of complete multigraphs into stars, Discrete Math. 26 (1979) 273-278.

doi:10.1016/0012-365X(79)90034-7

[40] M. Tarsi, Decomposition of complete multigraph into simple paths: nonbalanced handcuffied designs, J. Combin. Theory Ser. A 34 (1983) 60-70.

doi:10.1016/0097-3165(83)90040-7

[41] S. Tazawa, Decomposition of a complete multipartite graph into isomorphic claws, SIAM J. Algebraic Discrete Methods 6 (1985) 413-417. doi: $10.1137 / 0606043$

[42] M. Truszczyński, Note on the decomposition of $\lambda K_{m, n}\left(\lambda K_{m, n}^{*}\right)$ into paths, Discrete Math. 55 (1985) 89-96. doi:10.1016/S0012-365X(85)80023-6

[43] K. Ushio, S. Tazawa and S. Yamamoto, On claw-decomposition of complete multipartite graphs, Hiroshima Math. J. 8 (1978) 207-210. doi: $10.32917 / \mathrm{hmj} / 1206135570$

[44] S. Yamamoto, H. Ikeda, S. Shige-ede, K. Ushio and N. Hamada, On claw decomposition of complete graphs and complete bigraphs, Hiroshima Math. J. 5 (1975) 33-42. doi:10.32917/hmj/1206136782

Received 10 May 2017

Revised 7 December 2018

Accepted 7 December 2018 